\section{ONOMÁVAEIN}

Revista semestral de lingüística, filología y traducción

\title{
Para entenderse: algunas reflexiones para mejorar la comunicación entre personal médico y pacientes en el ámbito de la salud mental ${ }^{1}$
}

Fostering Understanding: Some Considerations to Improve Communication Between Patients and Healthcare Providers in Mental Health Settings

\section{Bruno Echauri Galván \\ Universidad de Alcalá de Henares \\ España}

\section{(c) $(i)$}




\section{Resumen}

El presente artículo busca subrayar algunos problemas fundamentales que el empleo de cierta terminología especializada puede crear en la transmisión-asimilación de información en el ámbito de la salud mental. Para ello, hemos dividido el proyecto en dos partes. La primera pretende sentar unas bases teóricas a partir de las cuales inferir la problemática que a nivel lingüístico pueden generar nuestros términos de estudio. En la sección posterior, estas dificultades se extrapolarán al campo de la traducción, a fin de proponer alternativas que puedan ayudar a solventarlas en un contexto interlingüístico. Nuestros objetivos últimos son, por un lado, concienciar a los lectores de las repercusiones (positivas y negativas) que el lenguaje puede tener sobre las personas que padecen un trastorno mental y, por otro, sentar las bases para traducciones más apropiadas que puedan derivar en una comunicación más fluida y adecuada entre pacientes y servicios médicos, tanto en entornos monolingües como en comunicaciones donde el inglés y el español sean las lenguas involucradas. Como punto final, se sugerirán futuras líneas de investigación y aplicación de estas directrices a disciplinas afines como la interpretación.

Palabras clave: terminología; salud mental; comunicación intercultural; traducción.

\section{Abstract}

This project seeks to highlight certain basic problems that the use of specific terminology may create in the provision and understanding of information in the Mental Health ambit. The paper has been divided into two parts. The first one presents different theoretical basis from which some linguistic difficulties may arise. The subsequent section extrapolates these issues to an interlinguistic scenario in order to formulate proposals that may be useful to solve them through translation processes. The final goals of this project are i) to raise awareness among readers of the positive and negative impact that language may have on those persons suffering mental disorders and ii) to provide the basis for more accurate translations that may result in a more fluent and effective communication among patients and healthcare services, either in monolingual or interlinguistic communicative environments where English and Spanish are the Ianguages involved.

In addition, we will suggest extending some of these guidelines to closely-related disciplines such as interpreting.

Keywords: terminology; mental health; intercultural communication; translation.

1 El autor quiere agradecer su apoyo al proyecto InterMed de la Universidad de Alcalá de Henares, financiado por el Plan Nacional de I+D+i del Gobierno de España. 


\section{o. Introducción}

Los problemas relacionados con la salud mental son, por lo general, cada vez más comunes en la sociedad actual. En 2001, se calculaba que más de 400 millones de personas en el mundo sufrían algún tipo de trastorno mental y la tendencia alcista ante lo que resta de siglo (Malvárez, 2007; Organización Mundial de la Salud, 2001) los consolida como uno de los principales problemas sanitarios a nivel global. Este aumento de la prevalencia debería venir acompañado de un crecimiento equivalente de la conciencia social con respecto a las implicaciones de estas afecciones, algo que parece no ser así (Arrillaga Arizaga y otros, 2004) y redunda en una comunicación menos efectiva entre médicos y afectados. A este respecto, el lenguaje es un elemento clave para la difusión e intercambio de información entre ambas partes, que presenta problemas y particularidades tanto a nivel intralingüístico como en la comunicación entre diferentes idiomas. En ese sentido, el presente artículo pretende subrayar parte de la problemática surgida del contraste entre el español y el inglés.

La traducción médica como conjunto cuenta con numerosos y detallados estudios (Fernández y Alarcón, 1998; Asrin, 2000; Montalt y González Davis, 2007...) en los que se aborda un amplio abanico de problemas, algunos de carácter general y otros más específicos, como la yuxtaposición de la terminología médica con la menos técnica, las discrepancias y predilecciones a la hora de nombrar distintas enfermedades, las variaciones en la ortografía de algunos nombres propios (Marsh, 1999), el impacto sobre la traducción de la variada etimología (árabe, grecorromana, anglosajona...) del vocabulario médico específico (McMorrow, 1998). Sin embargo, algunas ramas de la medicina como la salud mental aún no cuentan con un volumen tan relevante de literatura a sus espaldas que permita abordar parte de la problemática que genera la traducción en este contexto. En un interesantísimo prólogo a la versión española del capítulo V de la décima edición de la Clasificación Internacional de Enfermedades (Organización Mundial de la Salud, 1999; CIE-10 en adelante), se recogen muchos de los desafíos que la traducción de terminología específica entre estas lenguas presenta en el ámbito de la salud mental. Nuestro trabajo busca hacerse eco de la problemática lingüística que esta introducción subraya y, para tratar de lograrlo, lo hemos dividido en dos secciones perfectamente diferenciadas. En la primera, se pretende i) contrastar y ampliar alguno de los puntos señalados en el prólogo del capítulo $\checkmark$ de la CIE-10, ii) añadiendo problemas lingüísticos derivados del contraste entre las versiones española e inglesa (American Medical Association, 2012) de este manual y las de la cuarta edición del Manual Diagnóstico y Estadístico de los Trastornos Mentales (American Psychological Association, 2000; López-Ibor y Valdés Miyar, 2002; DSM-IV en adelante), iii) así como otros aspectos recogidos de otras fuentes que creemos pueden ser de interés. Recalcados los problemas, la segunda parte del artículo se propone adaptar esta terminología a las necesidades de los lectores menos expertos en la materia en un contexto interlingüístico, para lo que se propondrán algunas pautas que puedan resultar útiles al traductor a la hora de acercar conceptos importantes al paciente a través de un vocabulario más fácil de asimilar. Por ello, el criterio principal a la hora de escoger nuestros puntos de análisis ha sido la búsqueda de términos relativamente comunes que presentan ciertas particularidades en su aplicación en esta disciplina sanitaria, ya que lo que se pretende es orientar a los traductores hacia usos adecuados y, en general, presentar a todos los interesados algunos giros que el lenguaje se permite al entrar en el terreno de la salud mental. Esta labor de difusión nos parece relevante, habida cuenta de que un mayor conocimiento por parte del gran público de los distintos aspectos relacionados con los trastornos mentales es fundamental para crear un entorno social más concienciado, comprensivo y dispuesto a ayudar a quien pade- 
ce estas afecciones (Jorm, 2000). Del mismo modo, utilizar un vocabulario apropiado cobra especial relevancia en un campo tantas veces incómodo para los pacientes y en el que el temor a las etiquetas negativas permanece (Corrigan, 2004) y se perpetúa, en parte, a través del lenguaje (Gary, 2005). Dicho todo esto, damos paso al cuerpo de nuestro estudio.

\section{Algunos problemas terminológicos 1.1. Enfermos sin enfermedades}

"Enfermedad" es un término poco utilizado en la descripción de afecciones mentales, para las que se prefiere el empleo de la palabra "trastorno" (Real Academia Nacional de Medicina, 2012). En este sentido, el DSM-IV (2002: xxix) define los trastornos mentales del siguiente modo: "[...] síndromes, patrones comportamentales o psicológicos de significación clínica que aparecen asociados a un malestar, a una discapacidad o a un riesgo significativamente aumentado de morir o de sufrir dolor, discapacidad o pérdida de libertad. [...] Cualquiera que sea su causa, debe considerarse como la manifestación individual de una disfunción comportamental, psicológica o biológica”.

Tanto este manual como la CIE-10 hablan en todo momento de "trastornos mentales", reservando el término "enfermedad" para las disfunciones médicas asociadas. Esto no implica ni que existan diferencias fundamentales entre ambos conceptos ni que los trastornos "físicos" y "mentales" no estén directamente relacionados y sean difíciles de disociar en muchos casos. Sin embargo, ambos manuales justifican el empleo de dos términos a través de diversos motivos, a saber: i) la necesidad de establecer una nomenclatura que diferencie el trastorno principal de los asociados o causales, ii) favorecer la minuciosidad en la evaluación y iii) facilitar la comunicación entre los profesionales de la salud mental.

Otro término recogido en ambos manuales y que conviene analizar es "problema". Es impor- tante destacar que, en este contexto, se hace un uso muy amplio de la palabra, empleada tanto para hacer referencias generales a afecciones de la salud como para designar aquellos factores "psicosociales y ambientales que pueden afectar al diagnóstico, tratamiento y pronóstico" (López-Ibor y Valdés Miyar, 2002: 31) de los trastornos mentales. Entre estos aspectos se cuentan acontecimientos negativos, dificultades familiares o interpersonales, deficiencias sociales o de recursos y, en definitiva, cualquier problema relacionado con el contexto vital de la persona en cuestión. Todos son elementos a tener en cuenta durante la evaluación del paciente, pero estos "problemas" no podrían considerarse ni síntomas ni enfermedades relacionadas con el trastorno y es importante tener este matiz en cuenta.

Para cerrar este apartado, conviene señalar que, a lo largo del texto, hemos decidido utilizar "afección" como genérico para denominar cualquier problema de salud, obviando posibles matices diferenciadores con otros términos de su mismo campo semántico que sí analizaremos en otras palabras.

\subsection{Delirium, delirio y delirante}

La terminología relacionada con la palabra "delirio" es un área problemática en el campo de la salud mental. Estudios como el llevado a cabo por expertos médicos de distintos países (Morandi y otros, 2008) destacan la diversidad terminológica y la falta de consenso alrededor de muchos términos asociados a lo que comúnmente denominamos "delirio", derivados muchas veces de fenómenos como el mal empleo de la sinonimia o las traducciones de una lengua a otra. Por consiguiente, creemos importante intentar clarificar conceptos, aunque sea a un nivel técnico elemental como el de este artículo.

En el vocabulario médico empleado en español, existe una notable diferencia entre los tres términos que dan título al apartado. Contra- 
riamente a lo que ocurre en el habla coloquial, "delirio" es el menos utilizado (y utilizable) de los tres. Algunas de las razones son que esta palabra ni permite diferenciar entre delirio confusional y delirio esquizofrénico (Organización Mundial de la Salud, 1999) ni resuelve la confusión que supone su coexistencia con el término "delusión” (Casals Rispau, 2006). En consecuencia, la CIE-10 descarta casi por completo su uso (solo lo emplea en contadas ocasiones) mientras que el DSM-IV sí lo recoge con mayor frecuencia pero siempre fuera de la nomenclatura estandarizada.

Los dos términos restantes son los que ambos manuales utilizan para definir trastornos y síntomas específicos. Así, el delirium es un trastorno de la conciencia, la atención, la memoria y otras cogniciones sin una etiología específica, ya que puede ser provocado por distintos factores. Por contra, "delirante" es un término que suele emplearse en la construcción "idea delirante", que hace referencia a aquellas falsas creencias que no pueden rebatirse a través de argumentos lógicos (Castro García y otros, 2004) o, lo que es lo mismo, define a medias lo que en el lenguaje coloquial asociamos a delirio(s) como sustantivo común. La otra mitad del concepto lo conformarían las alucinaciones, definibles como "percepciones sensoriales subjetivas que ocurren sin estimulación del órgano sensorial relevante" (Noé Sebastián y otros, 2001: 677).

\subsection{Síntomas positivos}

Esta paradoja despertará inmediatamente las sospechas de los lectores y, desde luego, con razón. Si decimos que una persona sufre alucinaciones, se muestra hostil o padece un trastorno formal del pensamiento, ¿consideraríamos que se encuentra perfectamente? Seguro que no. sin embargo, en el ámbito de la salud mental y concretamente en el tratamiento de trastornos psicóticos, los tres síntomas mencionados anteriormente se consideran "síntomas positivos" (Moriana Elvira y otros, 2004). En este contexto, debemos disociar el término "positivo" de la idea de "bueno": los síntomas positivos son aquellos que reflejan procesos mentales anómalos a través de la exageración de una función o comportamiento del paciente, mientras que los negativos abarcarían tanto los déficits funcionales y comportamentales como la disminución de determinadas funciones psíquicas; entre estos últimos, se cuentan la inexpresividad, la pérdida de interés y emoción, la anhedonia, el alejamiento de las relaciones sociales... (Cortés Ruíz, 2009). Otra diferencia sustancial entre ambos radica en que, mientras los primeros pueden tratarse farmacológicamente, los segundos presentan una menor respuesta a la medicación y, por tanto, es más factible que perduren en fases residuales del trastorno (Mueser, 2007 [2002]: 611).

\subsection{Enfermos crónicos}

El uso de la palaba "crónico" es un campo delicado abierto a la confusión terminológica. Ante las connotaciones negativas que arrastra el uso de este término y de otros asociados como "cronicidad", en los últimos años se han buscado alternativas que permitan eliminar o, cuando menos, restringir el empleo de esta palabra. Así, se estableció, por ejemplo, la denominación Trastorno Mental Severo (TMS) para hacer referencia a los trastornos mentales graves de larga duración que conllevan distinto grado de discapacidad y disfunción social (Rodríguez González, 2003). Estos dos últimos aspectos componen en gran medida la carga estigmatizante del término "crónico", puesto que, como señalan Turón, Sánchez Faust y Grau (2011), todavía se tiende a asociar a los enfermos de este tipo con la inutilidad o la incapacidad a la hora de desempeñar ciertas tareas. Según el mismo artículo y a fin de evitar estos matices peyorativos, se prefiere el empleo de la palabra "persistente", adjetivo que ha ido ganando presencia en las escalas internacionales en los últimos años y cuyo uso se recoge tanto en nomenclaturas concretas como a nivel general. Sin embargo, no existe todavía un consenso en lo concerniente a este tema y 
ambos adjetivos coexisten actualmente en el vocabulario español empleado en el campo de la salud mental. Así, mientras la CIE-10 aboga por el empleo de "persistente", el DSM-IV prefiere el empleo del término "crónico" para referirse al mismo concepto.

\subsection{Pánico, ansiedad y angustia}

El uso de estos términos presenta algunos puntos de conflicto que conviene analizar. En primer lugar, es importante resaltar que, en el lenguaje empleado en salud mental, pasan a ser sinónimos muy próximos a pesar de las diferencias que existen entre ellos en el habla coloquial. De este modo, la terminología técnica actual acepta, por ejemplo, el empleo de términos como "crisis de pánico" o "crisis de angustia” como síntomas dentro de los cuadros clínicos denominados "trastornos de ansiedad" (Ivanovic-Zuvic, 2002). Del mismo modo, la CIE-10 considera "angustia" y "ansiedad" como sinónimos equivalentes al inglés anxiety. Sin embargo, el empleo de estos tres términos no es indistinto, y presenta varias diferencias y semejanzas en ambos manuales que creemos conveniente subrayar:

1) Tanto el DSM-IV como la CIE-10 traducen anxiety disorders como "trastornos de ansiedad".

2) La traducción del término panic presenta diferencias significativas. Así, la CIE-10 traduce panic disorder como "trastorno de pánico", mientras que el DSM-IV utiliza "trastorno de angustia”.

3) El punto anterior deriva en otra diferencia sustancial entre ambos manuales. Mientras la CIE-10 descarta el empleo de "angustia" en la nomenclatura estandarizada, el DSM-IV hace lo propio con la palabra "pánico". Estos términos no utilizados en los nombres oficiales sí se emplean, sin embargo, a la hora de describir síntomas o situaciones concretas. A continuación, presentamos dos ejemplos:
- "Durante un episodio depresivo grave, el enfermo suele presentar una considerable angustia o agitación..." (Organización Mundial de la Salud, 1999; descripción de un episodio depresivo grave sin síntomas psicóticos).

- "El miedo o la ansiedad pueden transformarse en intensos, con pánico ante la locura o la muerte" (López-Ibor y Valdés Miyar, 2002: 257).

\subsection{Grave, agudo y mayor}

Es importante señalar que estos tres términos no son intercambiables, sino que cada uno de ellos tiene unas connotaciones y aplicación bastante concretas. Examinémoslos uno por uno:

- Grave: se emplea en la gradación de la gravedad de la afección. A este respecto, tanto la CIE-10 como el DSM-IV tienden a clasificar los distintos trastornos mentales como leves, moderados o graves.

- Agudo: aplicado a una enfermedad o situación afín que comienza de manera brusca, cursa con manifestaciones clínicas intensas y evoluciona de forma relativamente rápida y breve (Real Academia Nacional de Medicina, 2012).

- Mayor: reservado a afecciones específicas; principalmente aplicado a la depresión.

Por supuesto, existen otros matices en su aplicación, pero, como punto de partida, creemos que esta es una clasificación adecuada para señalar las diferencias entre ellos. De este modo, un trastorno de estrés post-traumático sería agudo cuando los síntomas tuvieran una duración inferior a tres meses o crónico si fuese superior (Montt y Hermosilla, 2001); un trastorno obsesivo compulsivo podría considerarse grave, moderado o leve a partir de una evaluación psicológica que calibre la gravedad del trastorno, midiendo la presencia y el grado en el que se dan 
distintos síntomas (Ballesteros Montero y Ulloa Flores, 2011), y un trastorno depresivo se calificaría como mayor si se considerara entre grave y moderado, siendo los casos leves su contrapunto (Gómez-Restrepo y otros, 2004). Estos ejemplos demuestran que los tres adjetivos analizados no funcionan como sinónimos y prueban lo cuidadoso que hay que ser a la hora de emplearlos.

\section{Implicaciones para la traducción in- glés-español}

Hasta ahora, hemos descrito algunos problemas tanto intra- como interlingüísticos relacionados con la terminología asociada a los trastornos mentales, lo que nos ha servido también para señalar algunas de las particularidades que presenta la relación entre lenguaje y salud mental, un campo en el que tratar de tender puentes culturales y lingüísticos entre emisores y receptores supone enfrentarse a dificultades que van más allá de la complejidad terminológica y la asimetría a nivel de conocimientos entre las partes. Desgraciadamente, el peso que las etiquetas negativas tenían hace años sobre los pacientes y su entorno (Link y otros, 1987) sigue todavía presente (Corrigan, 2004), lo que genera un problema adicional en los afectados, que deben enfrentarse, además de a los síntomas propios del trastorno, a las repercusiones consecuencia del estigma, a menudo más constantes y permanentes que los primeros (López y otros, 2008). El lenguaje no ha sido, por supuesto, impermeable a estos prejuicios, que han cargado ciertas palabras y conceptos de matices negativos y lo han convertido en uno de los factores fundamentales en la construcción de estereotipos y etiquetas. Así, la lengua ha sido, en general y hasta ahora, utilizada muchas veces como "arma”, pero puede también convertirse en remedio si en lugar de agravar o perpetuar esta situación lo que se pretende es aliviarla. Siendo esta última nuestra intención, los siguientes párrafos buscan, dentro de las limitaciones a las que se ve sujeto el traductor, favorecer y mejorar la transmisión de información entre el ámbito médico y los afectados (directa o indirectamente) por algún trastorno mental. Por ello, las propuestas de nuestro trabajo se dirigen fundamentalmente a la traducción de documentos divulgativos, contexto dentro del cual intentaremos hallar puntos de encuentro entre las teorías que defienden emplear un lenguaje técnicamente apropiado, a fin de que el paciente se familiarice con conceptos clínicos específicos (Albin, 1998; Newmark, 1979), y las que hablan de adaptar el mensaje al vocabulario del lector para que comprenda fácilmente los conceptos que se le presentan (Herget y Alegre, 2009; Weiss, 2007). Esto implica buscar un delicado equilibrio entre pragmatismo y especificidad con el objeto de desarrollar un código formalmente adecuado que permita, al mismo tiempo, una transmisión precisa de información; para tratar de encontrarlo, analizaremos las opciones que la terminología estandarizada ofrece, buscaremos la alternativa más idónea en función de la audiencia de destino y/o aplicaremos estrategias traslativas como la generalización (Klaudy, 2003 en Kubáčková, 2009: 35), la normalización o la explicitación (Halverson, 2010: 351) —dentro de la cual incluimos el empleo de paráfrasis y explicaciones (Baker, 2011: 33-43) - sobre los puntos descritos en la sección anterior, estableciendo la idoneidad clínica de nuestras propuestas como principal frontera para estas prácticas. En esta línea, también buscaremos limitar la variedad terminológica a partir de la idea de Albin (1998) de que es conveniente crear un vocabulario concreto para cada texto, empleando palabras que el lector asocie a un único significado a fin de facilitar la asimilación del mensaje. Todo este proceso podría enmarcarse dentro de las competencias de los traductores que actúan como mediadores lingüístico-culturales en el ámbito sanitario (más detalles sobre esta figura en Castiglioni, 1997), a cuya complicada labor este proyecto pretende ayudar esbozando algunas pautas que permitan, dentro del campo de la salud mental, i) comunicar la misma idea ii) en un lenguaje preci- 
so pero también comprensible para el paciente, que además iii) reduzca en lo posible el empleo de ciertos términos "marcados" negativamente que pueden generar incomodidad en el lector. Antes de seguir, conviene, sin embargo, señalar que las propuestas recogidas a continuación son ejemplos de decisiones informadas (Casals Rispau, 2006) que creemos válidas y correctas, pero que admitirían el empleo de alternativas en función de factores como el contexto o el skopo de la comunicación, siempre que estas estuvieran adecuadamente justificadas.

\subsection{Enfermedad, trastorno y problema}

Resumiendo lo visto en el apartado 1.1, la mayor parte de disfunciones listadas tanto en el DSM-IV como en la CIE-10 se definen como "trastornos"; el término "enfermedad" se reserva para hacer referencia al resto de disfunciones médicas y a la palabra "problema" se le dan múltiples usos y significados, entre ellos el de definir las condiciones psicosociales o ambientales (laborales, económicos, de vivienda...) que pueden afectar al estado de salud del paciente. Esta diferenciación también se produce en inglés (siendo disorder, disease y problem los equivalentes empleados) y de ella podemos sacar distintas conclusiones de cara a la traducción. El error en el uso del término apropiado es relativamente fácil de solventar. Sin embargo, sí que es necesario tener en cuenta las líneas teóricas anteriores para escoger la opción apropiada. No obstante, si se desconoce o se ha olvidado la denominación correcta, podemos establecer una pauta general que favorezca la diferenciación entre los tres términos, limitando así la variedad terminológica y facilitando que el paciente se familiarice con el vocabulario utilizado, ya que los términos a recordar quedan semánticamente mejor acotados. Nuestra propuesta en este sentido coincide casi por completo con las decisiones adoptadas en los manuales y consistiría en utilizar el término "trastorno" cuando se hable de una afección relacionada con la salud mental y "enfermedad" para el resto de disfunciones médicas, dejando el empleo de "problema" para describir otro tipo de agravantes. De este modo, para un cuadro en el que el paciente sufra, por ejemplo, demencia por enfermedad de Parkinson acompañada por problemas sociales, "trastorno" sería el hiperónimo adecuado para "demencia” (dementia), "enfermedad" el que usaríamos para la enfermedad de Parkinson (Parkinson disease) y "problemas" el término apropiado para hacer referencia a esas circunstancias del entorno que influyen de manera negativa en el paciente (social problems).

\subsection{Delirium, delirio e ideas delirantes}

En el apartado 1.2 de este artículo describíamos cómo el principal problema en relación a estos términos surge del choque que se produce entre el uso común y clínico de la palabra "delirio". Como señalábamos, entre las personas ajenas al campo de la medicina, esta palabra es la más reconocible y utilizada de las tres, mientras que, desde un punto de vista clínico, parece la menos correcta y recomendable si tomamos en consideración el limitado uso que los manuales oficiales hacen de ella. El debate que se plantea a partir de esta situación es si seguir las directrices de estos últimos y descartarla o, por el contrario, defender su empleo al tratarse de un término fácilmente identificable por el gran público. Nuestra opinión en este caso coincide con la de los manuales diagnósticos y a continuación explicamos por qué.

La primera razón para descartar el empleo de la palabra "delirio" es, al igual que hicimos con "angustia", limitar la variedad terminológica: la inclusión de "delirio" en una traducción implica en la mayor parte de los casos añadir un término innecesario, puesto que contrastando las versiones inglesas y españolas de la CIE-10 y el DSM-IV comprobamos que las palabras empleadas son 
delirium en ambas lenguas para nombrar al trastorno propiamente dicho ${ }^{2}$ y delusion por "ideas delirantes". En otras palabras, utilizar "delirio" supone, desde un punto de vista puramente clínico, añadir a la comunicación un término sin un equivalente claro en otros idiomas que quedaría, por consiguiente, en un limbo semántico que resulta innecesario crear. Pero dejando a un lado los aspectos más técnicos, esta pauta también puede justificarse con argumentos más familiares para todos los que no somos expertos en salud mental. Un rápido análisis de la concepción general de "delirio" permite observar los matices peyorativos que el uso y el tiempo han dado a esta palabra. El diccionario de la RAE (2001), por ejemplo, la define como "acción de delirar", un verbo que, a su vez, arrastra connotaciones negativas desde sus orígenes, ya que se trata de un término derivado del latín delirare, que literalmente significa "salirse del surco" pero que se empleaba metafóricamente con el sentido de hacer locuras, extravagancias o estar fuera de los cabales (Corominas y Pascual, 1980). Este significado se ha conservado e incluso potenciado hasta llegar a nuestros días, donde la mayor parte de la gente sigue identificando este término con "decir, creer o pensar cosas disparatadas o incoherentes; concebir ilusiones insensatas" (María Moliner, 2012). Si restringimos o eliminamos el uso de la palabra "delirio" evitamos que estas enraizadas concepciones acudan a la mente del paciente durante la lectura, le ayudamos a familiarizarse con una nomenclatura más exacta y para nada compleja y, además, subsanamos otra inexactitud clínica presente en la última definición que citábamos, puesto que aceptarla implica considerar las ideas delirantes ("decir, creer o pensar cosas disparatadas o incoherentes") y las alucinaciones ("concebir ilusiones insensatas") como una misma cosa cuando no es así. Seguir la pauta que hemos marcado permite diferenciar también estos dos conceptos, empleando "alucinaciones" como traducción del término clínico hallucinations, y no "delirios", para englobar dos síntomas que es aconsejable diferenciar.

En resumen, en base a los argumentos presentados podemos sugerir que i) descartar el uso del término "delirio" en la traducción está generalmente justificado puesto que ii) el empleo de delirium para nombrar al trastorno y de iii) "ideas delirantes" y "alucinaciones" para la sintomatología iv) cubre con propiedad la nomenclatura anglosajona a través de términos comprensibles para el paciente y con una menor carga negativa.

\subsection{Síntomas positivos y negativos}

Si acudimos a los diccionarios de uso común, comprobamos cómo la definición de positivo ("cierto o real; práctico o provechoso" en el diccionario de María Moliner; "cierto efectivo, verdadero y que no ofrece duda; útil o práctico" en el de la RAE) a la que normalmente recurrimos difiere considerablemente del empleo que se hace de la palabra en este contexto. Por ello, en la traducción de documentos de carácter divulgativo, conviene indicar al lector a qué nos referimos con este término, por lo que, si no está explicado o no encontramos ninguna aclaración en el original, sería recomendable explicitar su verdadero significado a fin de evitar potenciales confusiones. Pero también es posible evitar la paráfrasis a través del uso de un término concreto.

Teniendo en cuenta que los términos en inglés también son positive y negative symptoms, su denominación oficial en este idioma tampoco ofrece ninguna pista o pauta que pueda dar lugar a una traducción menos confusa. Tratando de resolver el problema, encontramos que los términos "productivo" (productive symptoms) y "deficitario" (deficit symptoms) son considerados sinónimos muy próximos de "positivo" y

2 Al margen de los manuales clínicos, otros expertos (Morandi y otros, 2008) recomiendan el empleo de delirium como término de uso global (no solo en inglés y en español) para referirse a esta afección. 
"negativo", respectivamente (Campbell, 1989). Estas podrían ser, en principio, dos alternativas válidas, sobre todo teniendo en cuenta que "deficitario" recoge mejor, en nuestra opinión, el sentido de disminución de las funciones normales de los síntomas negativos. Sin embargo, cabe argumentar que el adjetivo "productivo" podría considerarse confuso, dadas sus connotaciones positivas en el habla habitual. En consecuencia, proponemos dos posibles soluciones para aquellos casos en los que evitar la paráfrasis a través de un término concreto derive en una comunicación más fluida:

- Síntomas no deficitarios: Considerando "deficitario" un término fácil de relacionar con "pérdida", añadir este adverbio de negación puede ayudar al paciente a diferenciar y comprender ambos conceptos a través de la contraposición.

- Síntomas por sobreestimulación: Esta opción busca facilitar la asociación de ideas apelando a las exageraciones en el funcionamiento normal que provocan los síntomas positivos.

Huelga decir que estos términos no están oficialmente reconocidos. Su empleo tiene un propósito aclaratorio, buscando acercar el concepto médico al paciente a través de una terminología menos ambigua que limite los malentendidos. Dicho esto, mantenemos que la mejor de las opciones suele ser explicar el término al paciente de la forma más clara posible, lo que facilita su familiarización con el concepto y refuerza la confianza del paciente, cara a futuras consultas médicas (Simpson y otros, 1991).

\subsection{Cronicidad y estigma}

La situación descrita en la sección 1.4 dibuja un panorama ambiguo que ha provocado, entre otras cosas, que existan trastornos con más de un nombre oficial reconocido. De este modo, mientras que en el DSM-IV leemos "trastorno delirante crónico", la misma afección en la CIE-10 recibe el nombre de "trastorno por ideas delirantes persistentes", lo que valida el empleo de "persistente" como sinónimo. Esta situación nos lleva al debate sobre cuál de los dos resulta más adecuado utilizar en un proceso de traducción. Para tratar de dar una respuesta razonada a esta pregunta vamos a considerar varios factores. En primer lugar, conviene señalar que en inglés lo que aquí denominamos "crónico" recibe el tratamiento de chronic, como en el caso de los "trastornos de tics motores crónicos”, cuyo equivalente en la versión americana del DSM-IV es chronic motor tic disorder. De este modo, la consulta de cualquier diccionario (médico o de uso habitual) probablemente nos conduciría a utilizar "crónico" en una traducción hacia el español. Sin embargo, la tendencia a evitar el uso de esta palabra en el ámbito de la salud mental es cada vez más generalizada en nuestro país, lo que hace que considerar otras opciones menos obvias sea algo necesario. Este sería el caso, por ejemplo, del chronic post-traumatic stress disorder (extraído de Bisson y otros, 2007), cuya traducción más literal (y reconocida) sería "trastorno por estrés postraumático (de tipo) crónico" pero que, siguiendo las directrices marcadas por la CIE-10, podríamos perfectamente traducir como "trastorno por estrés postraumático persistente" a fin de evitar emplear el término que hemos "marcado" como negativo.

Para aquellos casos en los que el adjetivo "crónico" se emplee con un carácter más general fuera de la propia denominación del trastorno, además del tantas veces repetido "persistente", podemos manejar otras opciones derivadas de aplicar un proceso traslativo de generalización al término. En ese sentido, ejemplos como "de larga duración" o "prolongado" serían aceptables y también suavizarían los matices negativos asociados a la cronicidad.

\subsection{Panic, anxiety y sus complicaciones}

Si en la sección 1.5 analizábamos la proximidad semántica entre "pánico", "ansiedad” y "angustia" dentro del ámbito clínico y veíamos las 
diferentes opciones que presenta la traducción de los términos anxiety y panic, en este apartado vamos a tratar de limitar las opciones, de cara a la comunicación con el paciente a través de un equilibrio entre el lenguaje técnico y el empleo de estos términos en el habla cotidiana.

Pese a que la CIE-10 considera casi sinónimos los términos "ansiedad" y "angustia", anteriormente hemos mostrado cómo ambos manuales coinciden en traducir anxiety disorders como "trastornos de ansiedad". Por tanto, parece que, desde un punto de vista clínico, existe un consenso más o menos extendido con respecto a la traducción de la forma inglesa. Para acabar de esclarecer posibles dudas a la hora de escoger entre las dos opciones principales que presenta el español, hemos decidido examinar la idoneidad de utilizar "ansiedad" en el habla cotidiana a través de la consulta de dos diccionarios de uso común como son el de la RAE (2001) y el Diccionario de Uso del Español (Moliner, 2012), comprobando que, en ambos casos, se recoge como sinónimo de "angustia" y como término asociado al campo de la medicina. Parece, pues, que esta palabra encuentra ese equilibrio entre terminología técnica y lenguaje cotidiano que tan difícil resulta conseguir y, por tanto, se presenta como una mejor opción a la hora de traducir anxiety.

Mayor problema surge, sin embargo, a la hora de encontrar acuerdo para la traslación de panic, puesto que del contraste entre el DSM-IV y la CIE10 no logra extraerse una pauta clara. Para tratar de esclarecer esta cuestión, acudimos, como en el punto anterior, a la búsqueda de un equilibro entre especificidad y un vocabulario más próximo al público en general. Una de las principales disimilitudes de matiz entre ambos términos podemos verla al contrastar estas dos definiciones, elaboradas a partir de la consulta de los dos diccionarios mencionados en el párrafo anterior:

- Pánico: Miedo extremo o terror provocado por el peligro de un amenaza inminente y que a menudo es colectivo y contagioso.
- Angustia: Intranquilidad con padecimiento intenso, por ejemplo, por la presencia de un gran peligro o la amenaza de una desgracia.

Ambas definiciones, aunque similares, presentan algunas diferencias significativas. El empleo de palabras como "terror", "miedo" o "extremo" ("grandísimo" es el adjetivo empleado por María Moliner) parece dar a "pánico" una carga semántica que implica mayor gravedad. Este rasgo coincide con el hecho de que tanto la CIE-10 como el DSM-IV parecen de acuerdo en señalar el pánico como un estado de crisis superior a, por ejemplo, la ansiedad, por lo que su uso en detrimento de angustia ayudaría a destacar ese matiz diferenciador. Otro argumento a favor del uso de "pánico" es el efecto que provoca en los pacientes puesto que, según la $\mathrm{ClE}$-10, estos sienten que este término describe mejor sus sensaciones. En consecuencia, podemos considerar que el empleo de este término como traducción del inglés panic resulta semánticamente más adecuado, tanto a la hora de describir una afección concreta como para hablar de síntomas característicos como un "ataque de pánico". Para justificar esta elección se puede también recurrir al argumento de la limitación terminológica, según el cual condensar dos conceptos similares (angustia y pánico) en un solo término (pánico) resultaría una práctica adecuada.

En resumen, las pautas que sugerimos en relación a este punto son:

1) "Ansiedad” por anxiety.

2) "Pánico" por panic.

3) Descartar o, cuando menos, restringir el uso de "angustia".

\subsection{Describiendo la gravedad del tras- torno}

En este punto, hay que ser cuidadoso con el falso amigo que supone severe, adjetivo empleado con mucha frecuencia en el ámbito médico anglosajón a la hora de determinar la gravedad 
de los trastornos mentales ${ }^{3}$ y que será conveniente someter a un proceso de normalización contextualizado a la hora de traducir. Otra palabra relacionada con la que hay que tener cuidado es serious, frecuentemente empleada como sinónimo del término anterior. Tanto en el inglés escrito como hablado, podemos encontrarnos con construcciones como severe mental disorder o serious mental disorder refiriéndose exactamente a lo mismo. Por poner un ejemplo, dos artículos como el de Fazel y Danesh (2002), por un lado, y el de Teplin (1990), por otro, tratan un tema muy similar como es el impacto de los trastornos mentales graves en la población reclusa o detenida, pero cada uno utiliza un adjetivo diferente para calificar estas afecciones. La traducción de estos términos ha generado una duplicidad similar en el español, puesto que es casi tan común encontrar la denominación "trastorno mental severo" (López y otros, 2005) como "trastorno mental grave" (López y Laviana, 2007). Bajo nuestro punto de vista, esta última opción resulta mucho más adecuada y a continuación explicamos por qué.

A la hora de traducir estos términos es importante mantener la cabeza fría y no dejarse llevar por primeros impulsos que deriven en "traducciones-reflejo" como "severo" y "serio", ya que no son las más idóneas. La primera resultaría inadecuada por no adaptarse semánticamente al contexto, ya que entre las acepciones de esta palabra (Real Academia de la Lengua Española, 2001) no se recoge el significado de gravedad y no debería emplearse en el ámbito sanitario (Navarro, 2004). La segunda podría considerarse algo más apropiada en este sentido, puesto que dicho adjetivo puede utilizarse para describir algo "grave, importante o de consideración" como una enfermedad. Sin embargo, en español contamos con el término "grave", quev como explicábamos en la sección 1.6, tanto el
DSM-IV como la CIE-10 tienden a utilizar a la hora de calificar la gravedad de los trastornos, lo que en principio haría innecesario recurrir a otras alternativas más cuestionables.

En cuanto al resto de términos vistos en el apartado 1.6, las traducciones más adecuadas podrían ser las siguientes:

1) Agudo $\leftrightarrow$ acute. Ejemplos:

“...dos subtipos: el trastorno por estrés postraumático agudo, en que los síntomas se inician durante los seis meses posteriores al trauma..." (Carvajal, 2002: 1).

"Persons developing acute post-traumatic stress disorder differ markedly in their processing of early steps..." (Rahe, 1993: 1).

2) Mayor $\leftrightarrow$ major. Ejemplos:

"Guía práctica clínica sobre el manejo de la depresión mayor en el adulto" (Grupo de Trabajo sobre el manejo de la Depresión Mayor en el Adulto, 2008).

"Major depression fact sheet" (National Alliance on Mental Illness, 2009).

Este último término presenta un matiz semántico interesante puesto que, como ya mencionábamos anteriormente, abarca los trastornos depresivos graves y moderados. Por tanto, en ciertas ocasiones no sería incorrecto utilizar el adjetivo "grave" siempre que estemos seguros de que este es el grado en el que la afección se manifiesta en el paciente. En este sentido, toda depresión grave es mayor, pero no toda depresión mayor puede considerarse grave.

Para finalizar este apartado, conviene señalar que los otros dos grados recogidos en la escala de gravedad de los trastornos, esto es, "leve" y "moderado", suelen traducirse (basándonos en la versión inglesa de la CIE-10 y el DSM-IV) como mild y moderate, respectivamente.

3 Además de utilizarse para describir la gravedad de los trastornos, severe también puede aparecer con otros significados en la comunicación en salud mental (por ejemplo, para gradar la intensidad de los síntomas). En estos casos, el abanico de posibilidades a la hora de traducirlo se abriría en función del contexto (Navarro, 2004). 


\subsection{Metonimia peyorativa}

En este último punto, sin equivalente en la sección 1 del artículo, hemos decidido describir un fenómeno lingüístico que, en nuestra opinión, juega un papel importante en la estigmatización y el etiquetado del paciente con un trastorno mental. Al igual que en otros ámbitos de la medicina no nos referimos a una persona como "sidosa" o "cancerosa", no parece adecuado definir a un paciente por su enfermedad en ningún otro contexto, incluido el de la salud mental (López-Ibor y Valdés Miyar, 2002: xxix). Así, resulta mucho más adecuado hablar de un paciente que "padece esquizofrenia" que de un "esquizofrénico" o de alguien que "sufre demencia" que de "un demente". El uso de estos nombres se ha ido cargando poco a poco de matices peyorativos, ya que el significado que han adquirido se basa en la conservación de los principales aspectos negativos de la afección. En consecuencia, tendemos a relacionar palabras como "esquizofrénico" o "psicótico" con los casos extremos con los que normalmente entramos en contacto a través del cine o la televisión. Esta visión prototípica y alejada de la realidad tiene su reflejo incluso en diccionarios como el de María Moliner (2012), donde en la definición de "demente" puede leerse "loco: se aplica a la persona que tiene la mente trastornada". Es difícil pensar que un traductor profesional se vea tentado de acortar frases como people with bipolar disorder go through unusual mood changes (National Institute of Mental Health, 2009) para escribir que "los bipolares experimentan cambios inusuales en su estado de ánimo ". Igualmente, resulta poco probable que profesionales de la sanidad utilicen la metonimia en este aspecto. Sin embargo, el uso de nombres como "esquizofrénico" (schizophrenic en inglés) está hoy en día bastante extendido, por lo que es factible que se filtren en la comunicación (especialmente en la oral) en algún momento. Por ello, si cualquiera de las partes involucradas emplease este fenómeno de cambio semántico, conviene esforzarse en corregirlo, sustituyendo este tipo de denominaciones por estructuras alternativas en las que se parafrasee el concepto como "paciente/persona con esquizofrenia" (patients/people with schizophrenia4), "paciente/persona que padece esquizofrenia" (patients/people suffering from schizophrenia4), "afectados por la esquizofrenia" (people affected by schizophrenia4), etcétera.

\subsection{Orientaciones hacia la interpreta- ción}

Como disciplina "hermanada" con la traducción, la interpretación es otro de los campos donde la comunicación intercultural tiene una especial importancia. Por ello, consideramos que sería posible extrapolar varias de las pautas expuestas hasta ahora a esta rama de la comunicación interlingüística.

Sin embargo, las directrices a seguir en esta disciplina varían inevitablemente, puesto que está expuesta a condicionantes distintos, como las restricciones de tiempo o la celeridad en la toma de decisiones inherente a la profesión. Estos rasgos generan una problemática particular que, por ejemplo, provoca que sea más fácil dudar entre el uso de una u otra denominación a la hora de trasladar un término de uno a otro idioma (como botón de muestra, basta imaginar una situación en la que la parte angloparlante utilice términos como disease, disorder o illness indistintamente y la consiguiente dificultad para el intérprete de trasladar el concepto con precisión).

Otro problema que puede surgir a la hora de interpretar es la dificultad de introducir explicaciones y aclaraciones terminológicas de cierta extensión. Incluso cuando fuera posible, la velocidad de la comunicación oral hace más difícil fijar y asimilar conceptos. Es por ello que, 
siguiendo con los ejemplos que hemos visto anteriormente, resultaría más positivo para la comunicación utilizar un término concreto en lugar de una paráfrasis, más sencilla de introducir en un texto escrito.

Estas y, por supuesto, otras cuestiones, abren, a mi modo de ver, una importante vía de investigación que, a través de estudios empíricos e indagaciones más exhaustivas, podrían llevar a interesantes conclusiones que seguro mejorarían las interacciones interculturales entre médico y paciente en el ámbito de la salud mental.

\section{Conclusiones}

El avance y aumento de la prevalencia de los trastornos mentales hace que cada vez sea más necesario familiarizarse con el vocabulario asociado a la salud mental. Sin embargo, este no es un campo sencillo, puesto que a la problemática inherente a toda terminología técnica o específica se unen rasgos distintivos delicados, como el peligro/miedo al estigma o la incomodidad con la que generalmente el paciente afronta cualquier comunicación relacionada con los trastornos de este tipo. Estas y otras razones convierten la salud mental en un entorno comunicativo particular con múltiples aristas y factores a tener en cuenta, más cuando existe una barrera lingüística, cultural y de conocimientos entre las partes implicadas. En ese sentido, lejos de querer aleccionar a los más expertos, la pretensión de este proyecto ha sido acercar a todo lector, pero en particular a los traductores que se adentran por primera vez en la materia, a algunos conceptos fundamentales y problemáticos, explicando, por un lado, su significado y razón de ser para, posteriormente, aplicar esta base de conocimiento a la comunicación entre distintas lenguas, en este caso, el español y el inglés. Teniendo en cuenta que las consideraciones recogidas en este artículo son solo parte de un todo mucho más complejo, nuestra intención es dar continuidad a esta línea de investigación incluyendo nuevos puntos de análisis y ampliando el estudio a disciplinas afines para seguir explorando los distintos ángulos del lenguaje en el campo de la salud mental, ajustar su uso a las necesidades de la comunicación entre los pacientes y el colectivo médico, y crear más y mejores propuestas que puedan ayudar a traductores y otros profesionales en la difícil labor de desarrollar estrategias que posibiliten una comunicación interlingüística más adecuada en este contexto.

\section{Agradecimientos}

A Sonia Martínez Castellano (enfermera especializada en salud mental) y Triana Sanz Font (psicóloga) por su asesoramiento y colaboración.

\section{Bibliografía citada}

AlBIN, Veronica, 1998: "Translating and formatting medical texts for patients with low literacy skills", Translation and Medicine (ed. Henry Fischbach). Amsterdam, Filadelfia: John Benjamins, 117-129.

American Medical Association, 2012: ICD-10-CM 2013: The Complete Official Draft Code Set, Chicago: Autor.

American Psychological Association, 2000: Diagnostic and statistical manual of mental disorders, 4th ed.: DSM-IV-TR, Washington, DC: Autor.

Arrillaga Arizaga, Maite, y otros, 2004: "Actitudes del personal sanitario de atención primaria hacia el enfermo mental, la psiquiatría y el equipo de salud mental", Revista Atención Primaria 33(9), 491-495.

AsRIN, Luis, 2000: Inglés médico: manual de traducción, Córdoba: El Galeno.

BAKer, Mona, 2011: In other words: A coursebook on translation, $2^{\text {nd }}$ edition. Londres, Nueva York: Routledge, Taylor \& Francis Group. 
Ballesteros Montero, Ana T., y Rosa E. Ulloa Flores, 2011: "Estudio comparativo de las características clínicas, demográficas y el funcionamiento familiar en niños y adolescentes con trastorno obsesivo-compulsivo leve a moderado vs. grave", Salud Mental 34(2), 121-128.

Bisson, Jonathan I., y otros, 2007: "Psychological treatments for chronic post-traumatic stress disorder. Systematic review and meta-analysis", British Journal of Psychiatry 190, 97-104.

CampbelL, Robert J., 1989: Psychiatric dictionary, sixth edition. Nueva York, Oxford: Oxford University Press.

Carvajal, César, 2002: "Trastorno por estrés postraumático: aspectos clínicos", Revista chilena de neuropsiquiatría 40(2), 20-34.

Casals Rispau, Sergi, 2006: "Reflexiones sobre la variación terminológica del español científico ilustradas con el caso del término inglés 'delusion'”, Panace@ 7(24), 222-227.

Castiglonon, Marta, 1997: La mediazione linguisticoculturale: Principi, strategie, esperienze, Milán: FrancoAngeli.

Castro García, Alfonso, y otros, 2004: "Complicaciones psiquiátricas de la enfermedad de Parkinson: clínica y tratamiento", Revista de Neurología 39(7), 646-650.

Corominas, Joan, y José A. Pascual, 1980: Diccionario crítico etimológico Castellano e Hispano, Madrid: Gredos.

Corrigan, Patrick, 2004: "How stigma interferes with mental health care", American Psychologist 59(7), 614-625.

Cortés Ruiz, María J., 2009: Endofenotipos disposicionales en los pacientes psicóticos y sus fami- liares de primer grado. Tesis doctoral, Universitat Rovira i Virgili.

Fazel, Seena, y John Danesh, 2002: "Serious mental disorder in 23000 prisoners: a systematic review of 62 surveys", Lancet 359, 545-550.

Fernández, Félix, y Esperanza Alarcón Navio, 1998: "La terminología especializada y el léxico común en el marco de la traducción de textos médicos de divulgación" en Leandro F. Fernández y E. Ortega Arjonilla (coords.): Traducción e interpretación en el ámbito biosanitario, Granada: Editorial Comares, 169-186.

Gary, Faye A., 2005: "Stigma: Barrier to mental health care among ethnic minorities", Issues in Mental Health Nursing 25(10), 979-999.

Gómez-Restrepo, Carlos, y otros, 2004: "Prevalencia de depresión y factores asociados con ella en la población colombiana", Revista Panamericana de Salud Pública 16(6), 378-386.

Grupo de Trabajo sobre el Manejo de la Depresión Mayor en el Adulto, 2008: Guía de Práctica Clínica sobre el Manejo de la Depresión Mayor en el Adulto, Madrid: Plan Nacional para el SNS del Ministerio de Sanidad y Consumo.

Halverson, Sandra, 2010: "Cognitive translation studies", Translation and Cognition (eds. Gregory M. Shreve y Erik Angelone). Amsterdam, Filadelfia: John Benjamins, 349-369.

Ivanovic-Zuvic, Fernando, 2002: "Diagnóstico diferencial de las crisis de angustia", Revista chilena de epilepsia 3, 18-28.

Jorm, Anthony F., 2000: "Mental Health literacy: Public knowledge and beliefs about mental disorders", British Journal of Psychiatry 177, 396-401.

KubÁČKovÁ, Jana, 2009: "Keeping Czech in check: A corpus-based study of generalization in transla- 
tion", SKASE Journal of Translation and Interpretation 4(1), 33-52.

LINK, Bruce G., y otros, 1987: "The social rejection of former mental patients: Understanding why labels matter", American Journal of Sociology 92(6), 1461-1500.

López, Marcelino, y Margarita LAVIANA, 2007: "Rehabilitación, apoyo social y atención comunitaria a personas con trastorno mental grave. Propuestas desde Andalucía", Revista de la Asociación Española de Neuropsiquiatría 27(1), 187-223.

LóPEz, Marcelino, y otros, 2008: "La lucha contra el estigma y la discriminación en salud mental. Una estrategia compleja basada en la información disponible", Revista de la Asociación Española de Neuropsiquiatría 28(101), 43-83.

López, Marcelino, y otros, 2005: "Evaluación del Programa residencial para personas con trastorno mental severo en Andalucía (I): descripción general del programa y del estudio", Rehabilitación psicosocial 2(1), 2-15.

López-Ibor, Juan J., y Manuel Valdés Miyar, 2002: Manual diagnóstico y estadístico de los trastornos mentales, $4^{a}$ ed.: DSM-IV-TR, Barcelona: Masson.

Malvárez, Silvina, 2007: "El reto de cuidar en un mundo globalizado", Texto \& Contexto Enfermería 16(3), 520-530.

McMorrow, Leon, 1998: "Breaking the Greco-Roman mold in medical writing: the many languages of the $20^{\text {th }}$ century medicine" en Henry FischBaG: Translation and Medicine, Amsterdam/Philadelphia: John Benjamins Publishing Company, 13-29.

Moliner, María, 2012: Diccionario de uso del español, Madrid: Gredos.

Montalt, Vincent y Gonzalez Davis, María, 2007: Medical translation step by step: learning by draf- ting, Manchester, UK Kinderhook, New York: St. Jerome Publishing.

Montt, María E., y Wladimir Hermosilla, 2001: "Trastorno de estrés post-traumático en niños”, Revista Chilena de Neuropsiquiatría 39(2), 110-120.

Morand, Antonio, y otros, 2008: "Understanding international differences in terminology for delirium and other types of acute brain dysfunction in critically ill patients", Intensive Care Med 34(10), 1907-1915.

Moriana Elvira, Juan A., y otros, 2004: "Tratamiento combinado de la esquizofrenia aplicado en el ámbito domiciliario", Psicothema: Revista Anual de Psicología 16(3), 436-441.

Mueser, Kim T., 2007 [2002]: "Tratamiento cognitivo-conductual de la esquizofrenia" en Manual para el tratamiento cognitivo-conductual de los trastornos psicológicos, vol. $1,2^{a}$ ed. (dir. Vicente E. Caballo), Torrejón de Ardoz: España, 611-633.

Navarro, Fernando A., 2004: "Severe: el debate de nunca acabar", Apuntes 12(1), 16-18.

Newmark, Peter, 1979: "A layman's view of medical translation”, British Medical Journal 2, 1405-1407.

Noé Sebastián, Enrique, y otros, 2001: "Trastornos neuropsiquiátricos en la enfermedad de Parkinson”, Revista de Neurología 32(7), 676-681.

RAHE, Richard H., 1993: "Acute versus chronic posttraumatic stress disorder", Integrative physiological and behavioral science 28(1), 46-56.

Real Academia de la lengua Española, 2001: Diccionario de la Lengua Española, $22^{a}$ edición, Madrid: Espasa.

Real Academia Nacional de Medicina, 2012: Diccionario de Términos Médicos, Madrid: Editorial Médica Panamericana. 
Rodriguez GonzAlez, Abelardo, 2003: “Organización de cuidados comunitarios el papel de la atención psicosocial y social en la atención comunitaria a las personas con enfermedad mental grave y crónica" en Atención comunitaria, rehabilitación y empleo: Il Congreso de Rehabilitación en Salud Mental (coords. Miguel A. Verdugo Alonso y otros), Salamanca: INICO, 67-95.

Simpson, Michael, y otros, 1991: "Doctor-patient communication: the Toronto consensus statement", British Medical Journal 303(6814), 13851387.

TePLIN, Linda A., 1990: "The prevalence of severe mental disorder among male urban jail detainees: comparison with the Epidemiologic Catchment Area Program", American Journal of Public Health 80(6), 663-669.

Turón, Vicente, y otros, 2011: "Atención a la cronicidad en los Trastornos de la Conducta Alimentaria”, trabajo presentado en Interpsiquis $12^{\circ}$ Congreso Virtual de Psiquiatría y Neurociencias.

WeIss, Barry D., 2007: Health literacy and patient safety: Help patients understand. Manual for clinicians, $2^{\text {nd }}$ edition, Chicago: American Medical Association.

\subsection{Referencias electrónicas}

Herget, Katrin, y Teresa Alegre, 2009: "Translation of medical terms", Translation Journal 3(13) [http:// www.bokorlang.com/journal/49medicalı.htm, fecha de consulta: 25 de enero, 2013].

Marsh, Malcolm, 1999: "Algunas consideraciones sobre la traducción médica" en Antonio Gı de Carrasco y Leo Hickey: Aproximaciones a la traducción [http://cvc.cervantes.es/lengua/aproximaciones/, fecha de consulta: 03 de septiembre, 2012].
National Alliance on Mental Illness, 2009: Major depression fact sheet [http://www.nami.org/ Template.cfm?Section=Depression\&Templa te=/ContentManagement/ContentDisplay. cfm\&ContentID=88956, fecha de consulta: 22 de enero de 2013].

National Institute of Mental Health, 2009: Bipolar Disorder (easy to read) [http://www.nimh.nih.gov/ health/publications/bipolar-disorder-easy-toread/nimhbipolardisorderbrochure.pdf, fecha de consulta: 21 de enero de 2013].

Organización Mundial de la Salud, 1994: The ICD-10 classification of Mental and Behavioural Disorders: Clinical descriptions and diagnostic guidelines [http://www.who.int/classifications/icd/ en/bluebook.pdf, fecha de consulta: 10 de enero de 2013]

Organización Mundial de la Salud, 1999: Edición Española del Capítulo V sobre Trastornos Mentales y del Comportamiento de la $10^{a}$ edición de la Clasificación Internacional de Enfermedades [http:// feafes.org/publicaciones/TrastornosmentalescomportamientoCIE10.pdf, fecha de consulta: 9 de enero de 2013].

Organización Mundial de la Salud, 2001: Por la salud mental en el mundo: sí a la atención, no a la exclusión [http://www.who.int/mental_health/ media/en/391.pdf, fecha de consulta: 28 de enero de 2013]. 